\title{
Polyaniline/AgCl Hybrid Materials for Selective Determination of Dopamine by Electrochemical Methods
}

\author{
Shuangli Zhou, Manyuan Xie, Xiaoli Yuan, Fulong Zeng, Wujun Zou, Dingsheng Yuan* \\ Department of Chemistry, Jinan University, Guangzhou, China \\ Email: tydsh@jnu.edu.cn
}

Received March 12, 2012; revised March 26, 2012; accepted April 25, 2012

\begin{abstract}
A promising electrochemical sensor based on PANI/AgCl hybrid material has been developed. The organic/inorganic hybrid material has exhibited good electrocatalytic properties by cyclic voltammetry measurement and differential pulse voltammetry. The oxidation overpotential of dopamine decreased dramatically, and the oxidation peak current increased significantly at PANI/AgCl/GCE compared to those obtained at PANI/GCE, $\mathrm{AgCl} / \mathrm{GCE}$ and bare GCE, corresponding to the synergistic effect between PANI and inorganic particle $\mathrm{AgCl}$. Under the optimized conditions, the linear response in the concentration range of 0.7 to $6.0 \mu \mathrm{M}$ for the selective determination dopamine on the $\mathrm{PANI} / \mathrm{AgCl} / \mathrm{GCE}$ is obtained with a detection limit of $5.4 \times 10^{-8} \mathrm{M}(\mathrm{S} / \mathrm{N}=3)$ using differential pulse voltammetry. The results indicated that the modified electrode can be used to determine dopamine without the interference from ascorbic acid and ensure high sensitivity and good selectivity.
\end{abstract}

Keywords: Polyaniline/AgCl Hybrid Material; Sensor; Dopamine; Selective Determination; Differential Pulse Voltammetry

\section{Introduction}

In recent years, the design, fabrication and application of novel sensors has been considerable interest. Particularly, the development of electrochemical sensors has been widely studied as a reliable method to quantitatively detect a large number of organic substances of biological importance, including hydrogen peroxide [1], hydroquinone [2], nicotinamide-adenine dinucleotide (NADH) [3], serotonin [4], glucose [5], dopamine (DA) [6] and ascorbic acid (AA) [7], and so on. Dopamine, the most significant catecholamine, belongs to the family of neurotransmitters and attracts considerable research in clinical research because of its involvement in the immune system, metabolic processes, and emotional processes. Several neurological disorders, in particular schizophrenia and Parkinsonism, are associated with the low concentration of DA [8-10]. DA is electrochemically active and electrochemically oxidized characteristic, which has long been a great deal of developments in electrochemical methods. However, there are some problems in electrochemical methods due to the nature of the electrooxidative reaction, involving two major problems in monitoring dopamine are selectivity and sensitivity. It is well known that the direct electrochemical oxidation of other neurochemical compounds, including AA and uric acid (UA),

${ }^{*}$ Corresponding author. is irreversible and always overlapping voltammetric response with each other at bare glassy carbon electrode (GCE), which results in rather poor selectivity and reproducibility. Moreover, the basal concentrations of DA and AA changes in a broad range from $1.0 \times 10^{-7}$ to $1.0 \times 10^{-3}$ $\mathrm{M}$ and the concentration of $\mathrm{AA}$ is usually 1000 times higher than that of DA in human brain. Quantitative determination of DA is therefore important and has been a subject of considerable interest between neuroscientists and chemists. Therefore, both sensitivity and selectivity are highly desirable for real analytical applications and diagnostic researches. To meet these needs, considerable strategies have been devoted to modify the electrode surface by using new electrode materials to obtain polymer-modified electrode or self-assembled electrode [1115], then the modified electrode can not only decrease the oxidation overpotential but also increase the sensitivity.

The strong $\pi-\pi$ conjugated macromolecules such as polyaniline (PANI), polypyrrole (PPY), and polythiophene (PTH) belong to one of the most interesting classes of conducting polymers (CPs). Since their discovery in the 1970s, they hold great promise for applications as electrochemical sensors due to the considerable flexibility in their chemical structures and their redox characteristics. Among the various types of CPs, PANI has many attractive features owing to its unique conduction mechanism, easy proton doping, readily film-forming ability, favo- 
rable environmental stability and having extensive applications in molecular electronic devices, fuel cells, nonlinear optics, sensors, etc. [16-20]. In particular, PANI can be used to construct selective electrode of sensors for DA determination since it can improve the electrocatalytic properties and increase the electron transfer rate and stability of the electrode response [21-24]. However, the drawbacks such as the poor selective and overlapping of the voltammetric response to DA are the limiting factor for the usage of CPs, and PANI combined with inorganic particles could overcome these limitations. Indeed, polymer-inorganic hybrid materials can keep the integrity and activity of inorganic as well as exhibit the unique conducting properties of polymer because of the strong synergistic effect between the inorganic particles and polymer matrixes. Yan and co-workers [25] synthesized AgCl@PANI coreshell structured nanocomposites and studied the electrocatalytic activities toward oxidation of $\mathrm{DA}$ and $\mathrm{AA}$ at $\mathrm{pH}$ neutral environment.

Although the composites based on PANI and $\mathrm{AgCl}$ have been reported, the selectivity and electrocatalytic activity for the composites with nanostructure is still a novel challenge. So far, dramatic efforts have been dedicated to developing new methods for the fabrication of polymer-inorganic hybrid materials. Herein, we have utilized a facile approach to synthesize conducting PANI nanowire networks immobilizing $\mathrm{AgCl}$ nanoparticles in the presence of polyvinypyrrolidone (PVP), and then coated the PANI/AgCl hybrid materials on the GCE to develop a sensitive and selective biosensor for DA determination. In addition, the modified electrode not only exhibited strong electrocatalytic activity toward the oxidation of DA and AA, but also successful separated their electrochemical responses in mixed solution. The separation of the oxidation peak potential for DA and AA as larger as $262 \mathrm{mV}$ by differential pulse voltammetry (DPV).

\section{Experimental}

\subsection{Chemicals and Solutions}

Aniline was obtained from Aldrich and purified twice by distillation under reduced pressure. Dopamine hydrochloride, ascorbic acid and potassium biiodate $\left(\mathrm{KH}\left(\mathrm{IO}_{3}\right)_{2}\right)$ were purchased from Aladdin Chemistry Co, Ltd. Nafion was obtained from Aldrich. N, N-dimethylformamide (DMF), $\mathrm{AgNO}_{3}, \mathrm{PVP}$, sodium phosphate monohydrate $\left(\mathrm{NaH}_{2} \mathrm{PO}_{4} \cdot \mathrm{H}_{2} \mathrm{O}\right)$ and sodium phosphate dibasic dehydrate $\left(\mathrm{Na}_{2} \mathrm{HPO}_{4} \cdot 2 \mathrm{H}_{2} \mathrm{O}\right)$ used for the preparation of PBS with various $\mathrm{pH}$ values were received from Guangzhou Chemical Reagent Factory (Guangzhou, China). Freshly prepared solutions of DA and AA were used in all the experiments. All other chemicals used were of analytical grade without further purification. Highly purity nitrogen was used for the deaeration. The solutions were prepared with double-distilled water.

\subsection{Apparatus}

The morphology was observed via a Philips TECNAI-10 transmission electron microscope (TEM) using an acelerating voltage of $100 \mathrm{kV}$. The functional groups on the surface of samples were detected via Nicolet FT-IR 6700 spectrometer with $\mathrm{KBr}$ plate in the $800-4000 \mathrm{~cm}^{-1}$ region. Ingsens electrochemical workstation (Ingsens, China) was used for the electrochemical measurements. A conventional three-electrode system was used throughout the experiments. The working electrode was a glassy carbon electrode, the auxiliary electrode was a platinum wire and an $\mathrm{Ag} / \mathrm{AgCl}$ (in saturated $\mathrm{KCl}$ solution) electrode as the reference electrode, respectively. All the potentials reported were referred to the $\mathrm{Ag} / \mathrm{AgCl}$ electrode.

\subsection{Preparation of PANI/AgCl Hybrid Materials}

For preparing $\mathrm{PANI} / \mathrm{AgCl}$ hybrid materials, $\mathrm{AgCl}$ and aniline were employed as raw materials and PVP as a template according to a reported procedure $[26,27]$ with some modifications. Typically, $1 \mathrm{mM}$ of $\mathrm{AgNO}_{3}$ and 1 $\mathrm{mM}$ of aniline were added respectively to $4 \%$ PVP aqueous solution. $20 \mathrm{~mL}$ of $1 \mathrm{M} \mathrm{HCl}$ aqueous solutions was dropped into the above mixture with magnetic stirring at room temperature for $2 \mathrm{~h}$. After that, a $100 \mathrm{~mL}$ of potassium biioodate $\left(0.012 \mathrm{M}, \mathrm{KH}\left(\mathrm{IO}_{3}\right)_{2}\right)$ was added to the solution which was magnetically stirred for $15 \mathrm{~min}$, and then sodium hypochlorite $(5 \%, \mathrm{NaClO})$ and the hypochlorite $(5 \mathrm{~mL}, \mathrm{HClO})$ was added and maintained for 20 min without stirring. The polymerization reaction was carried out under static condition at room temperature for $24 \mathrm{~h}$. Then the green suspension was filtered and continuously washed with $\mathrm{HCl}(1 \mathrm{M})$ until the filtrate became colorless, and then resultant precipitate was dried in oven at $60^{\circ} \mathrm{C}$ for $24 \mathrm{~h}$.

\subsection{Preparation of the PANI/AgCl Hybrid Materials Modified Glassy Carbon Electrode}

Prior to the surface modification, the bare GCE $(4 \mathrm{~mm}$ in diameter) was first carefully polished with $1.0,0.3$, and $0.05 \mu \mathrm{m}$ aluminum oxide $\left(\mathrm{Al}_{2} \mathrm{O}_{3}\right)$ powder, then rinsed with double-distilled water and ethanol in sequence, and finally was sonicated for 5 minutes. A mixture containing $3 \mathrm{mg}$ of PANI/ $\mathrm{AgCl}$ hybrid composites, $3 \mathrm{~mL}$ DMF solution was dispersed ultrasonically for $2 \mathrm{~h}$ to obtain a well-dispersed ink. A $5 \mu \mathrm{L}$ of above solution was then dropped on the surface of the pretreated GCE and then $10 \mu \mathrm{L} 1 \%$ Nafion was coated on it, then the electrodes dried under infrared lamp for $20 \mathrm{~min}$ to obtain the PANI/ $\mathrm{AgCl} / \mathrm{GCE}$. After modification, the electrodes were activated in $0.1 \mathrm{M}$ PBS solution at $\mathrm{pH} 7.3$ by cyclic scan- 
ning between -0.6 and $+1.5 \mathrm{~V}$ at the scan rate of 100 $\mathrm{mV} \cdot \mathrm{s}^{-1}$ for 20 cycles. Before and after each experiment, the modified electrodes were carefully washed with distilled water and reactivated by the above-mentioned method. All experiments were carried out in $10 \mathrm{~mL} \mathrm{pH}$ 4.0 PBS solutions at room temperature and all solutions were deaerated and purged by purity nitrogen before each experiment.

\section{Results and Discussion}

\subsection{Characterization of the PANI/AgCl Hybrid Composites}

Potassium biiodate, $\mathrm{KH}\left(\mathrm{IO}_{3}\right)_{2}$, is a primary standard substance that can be used as an oxidant. $\mathrm{KH}\left(\mathrm{IO}_{3}\right)_{2}$ decomposes according to the following reaction [27]:

$$
\begin{gathered}
\mathrm{KH}\left(\mathrm{IO}_{3}\right)_{2} \rightarrow \mathrm{K}^{+}+\mathrm{H}^{+}+2 \mathrm{IO}_{3}^{-} \\
2 \mathrm{IO}_{3}^{-}+12 \mathrm{H}^{+}+10 \mathrm{e}^{-} \rightarrow \mathrm{I}_{2}+6 \mathrm{H}_{2} \mathrm{O}
\end{gathered}
$$

In this experiment, potassium biiodate and sodium hypochlorite are used as co-oxidants. Figure 1 shows the typical TEM image of PANI/AgCl hybrid composites. The as-synthesized PANI is interconnected threadlike network, being wrapped the dark particles are $\mathrm{AgCl}$ nanoparticles. It can be seen that $\mathrm{AgCl}$ particles are successfully inserted into the PANI. Due to this special structure, the interfacial affinity of inorganic $\mathrm{AgCl}$ with PANI improves the electrochemical performance/the electrical contact due to tight space contact, which will be certified by further electrochemical measurement.

Figure 2 gives FI-IR spectrum of the PANI/AgCl hybrid material. Two absorption peaks in the IR spectrum of the sample at 3233 and $3436 \mathrm{~cm}^{-1}$ are attributed to the

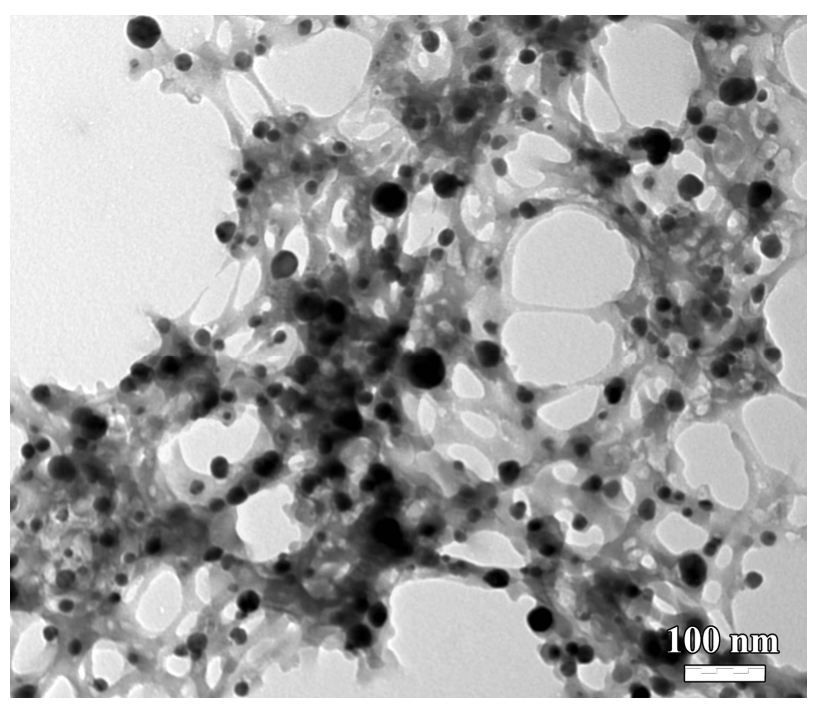

Figure 1. TEM image of PANI/AgCl hybrid composites synthesized with potassium biiodate co-used sodium hypochlorite as co-oxidant.

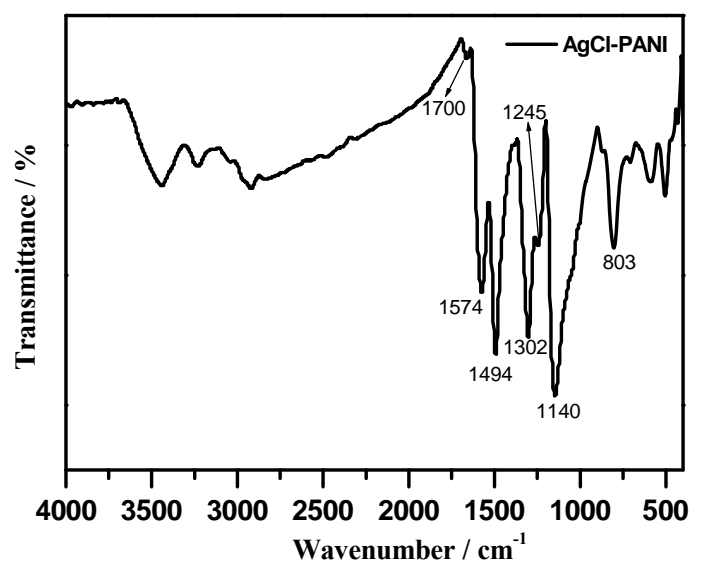

Figure 2. FT-IR spectrum of PANI/AgCl composite.

stretching $-\mathrm{OH}$ and $-\mathrm{NH}$ modes arising from superficial adsorption water and PANI, respectively. The small peaks at 2922 and $2851 \mathrm{~cm}^{-1}$ are originated from the benzenoid ring stretching vibration of $\mathrm{C}-\mathrm{H}$ bond, and the characteristic bands at 1574 and $1494 \mathrm{~cm}^{-1}$ attribute to $\mathrm{C}=\mathrm{C}$ a stretching deformation mode of the quinoid and benzenoid rings. Bands at 1302 and $1245 \mathrm{~cm}^{-1}$ are related to the $\mathrm{C}-\mathrm{N}$ stretching of the secondary aromatic amine, $\mathrm{C}-\mathrm{N}$ stretching vibration in the polaron structure, respectively. Bands at 1140 and $803 \mathrm{~cm}^{-1}$ are the results of a vibration mode of the $\mathrm{N}=\mathrm{Q}=\mathrm{N}$ stretching mode and an aromatic $\mathrm{C}-\mathrm{H}$ out-of-plane bending of 1,4-disubstituted benzene ring $[7,28]$. The absorption band assignable to $\mathrm{C}=\mathrm{O}$ is observed at $1700 \mathrm{~cm}^{-1}$, which indicated the presence of PVP.

\subsection{Electrochemical Behaviors of DA and AA at the PANI/AgCl/GCE}

Figure 3 gives the CVs in three different electrolytes at bare GCE, AgCl/GCE, PANI/GCE and PANI/AgCl/GCE in a $\mathrm{pH}$ 4.0 PBS solution. As can be seen from Figure 3(a), a pair of board redox peaks at PANI/GCE is observed, which is attributed to redox process normally found for the PANI system under weak acidic condition. Under the identical conditions, only a very weak current response can be observed at the $\mathrm{AgCl} / \mathrm{GCE}$ and bare $\mathrm{GCE}$. However, when $\mathrm{AgCl}$ nanoparticles are added into PANI matrix, the current response of $\mathrm{PANI} / \mathrm{AgCl} / \mathrm{GCE}$ is visibly reducing and the deviation of redox peaks becomes narrow. These changes indicate that PANI/AgCl/GCE will be advantageous for the detection of AA and DA.

Since AA is the major interfering substance in the electrochemical measurement of $\mathrm{DA}, \mathrm{CV}$ behavior of AA at modified electrode was also studied, as shown in Figure 3(b). Oxidation of $\mathrm{AA}$ at the bare $\mathrm{GCE}, \mathrm{AgCl} /$ GCE and PANI/GCE are generally believed to be totally irreversible, indicating the slow electron transfer kinetics of the electrodes. Compared to $\mathrm{CV}$ of PANI/AgCl/GCE 
in Figure 3(a), a new pair of redox peaks is presented, indicating the oxidation and reduction of AA.

Figure 3(c) shows the CVs of DA at different modified electrodes. Two well-defined redox peaks with the anodic and cathodic peak potentials of oxidation DA appear at 386 and $328 \mathrm{mV}$ are observed at the PANI/ $\mathrm{AgCl} / \mathrm{GCE}$, respectively. As peak-to-peak separation $(\triangle$ $E p)$ is a function of electron transfer rate, the lower $\triangle$ $E$ p, the faster electron transfer rate. The anodic peak potential shifted negatively value and the cathodic peak potentials shifted positively compared with that at the bare GCE. So the small $\triangle E p$ suggests that the reversebility of DA is considerably improved at $\mathrm{PANI} / \mathrm{AgCl} /$ GCE. A comparison between the anodic and cathodic peaks for $\triangle E \mathrm{p}$ is $58 \mathrm{mV}$ for $\mathrm{PANI} / \mathrm{AgCl}$ modified electrode and $78 \mathrm{mV}$ for the PANI/GCE in $1 \mathrm{mM} \mathrm{DA}$. Compare to the blank PBS solution, the anodic peak current at $\mathrm{PANI} / \mathrm{AgCl} / \mathrm{GCE}$ is enhanced by about 2.8 times as well as the $\triangle E \mathrm{p}$ is decreased in comparison with the PANI/ GCE, implying the synergic effect of both the PANI organic polymer and inorganic particle in the hybrid composites at $1 \mathrm{mM} \mathrm{DA}$ solution. Therefore, the PANI/AgCl/ GCE has an electrocatalytic activity for DA oxidation in $0.1 \mathrm{M}$ PBS solution.
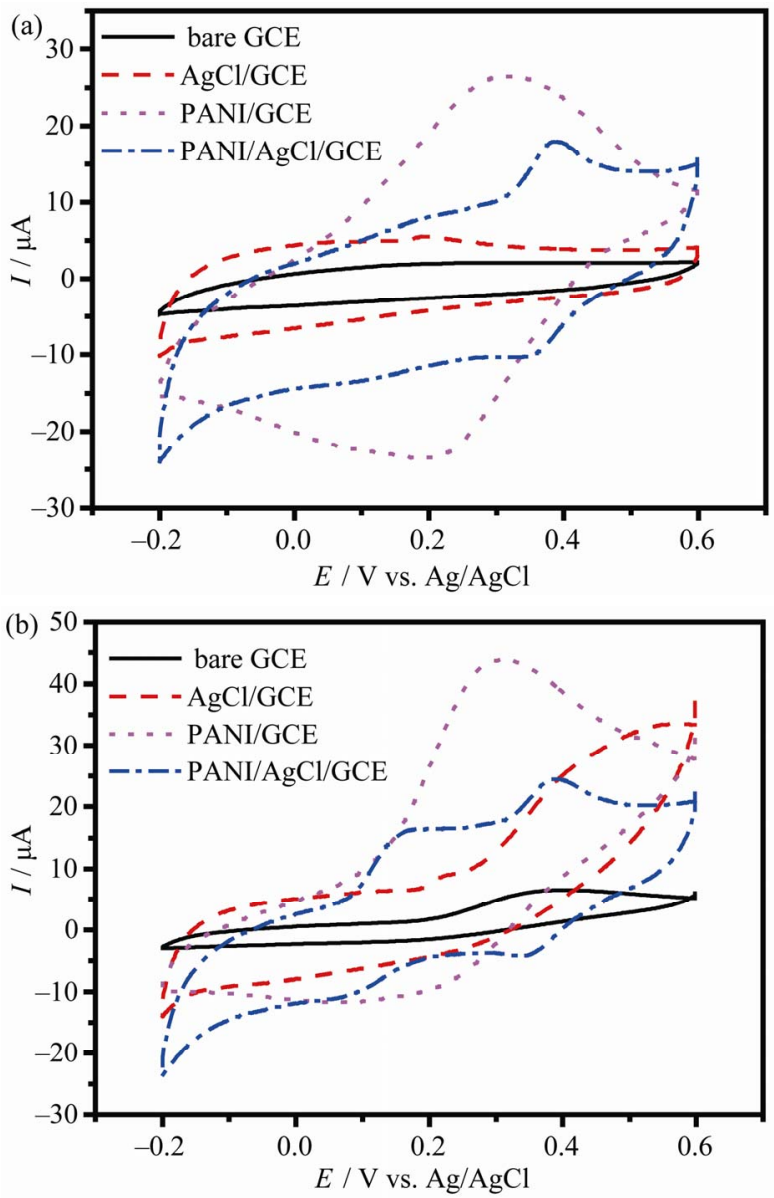

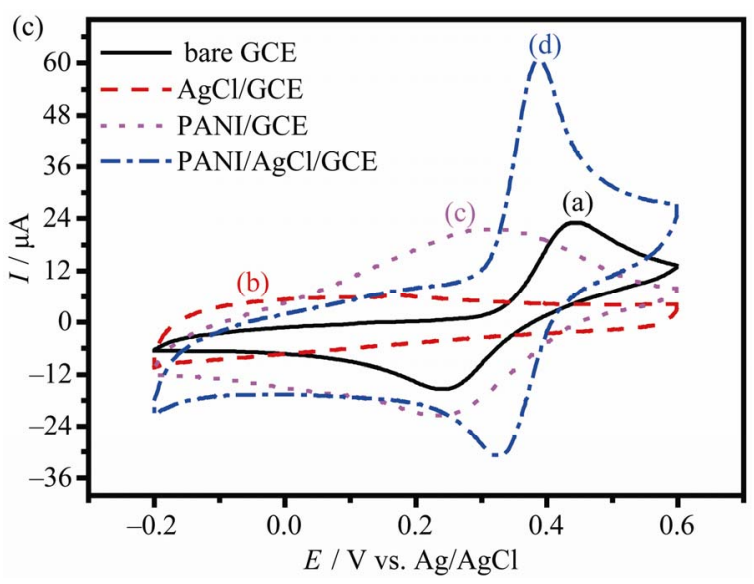

Figure 3. CVs for the bare GCE, AgCl/GCE, PANI/GCE and AgCl-PANI/GCE at $0.1 \mathrm{M}$ blank PBS (a), $1 \mathrm{mM}$ AA (b) and $1 \mathrm{mM} D A$ (c) in $\mathrm{PBS}$ solutions at $\mathrm{pH}=4.0$, respectively. Sweep rate: $50 \mathrm{mV} \cdot \mathrm{s}^{-1}$.

It is well known that silver halides are one type of important semiconductor. Although $\mathrm{AgCl}$ particles do not make a continuous electron path, the incorporated conducting $\mathrm{AgCl}$ generates more active sites for the charge transfer through the interface inside the electrode by making good contacts with the PANI matrix, thus PANI particles located far from the electrode surface can effectively take part in the redox reaction [26]. Under the identical conditions, Compare to curve (b), (c) and (d) in Figure 3(c), the peak current was significantly increased and overpotential was lower of DA at $\mathrm{PANI} / \mathrm{AgCl} / \mathrm{GCE}$ owing to the composite effect. The synergic effect of the catalytic system can be described as the accumulation ability of the PANI organic polymer which is enhanced by the inorganic nanoparticle of the $\mathrm{AgCl}$ in the hybrid composites.

\subsection{Effects of the Scan Rate and pH on Peak Currents of DA and AA}

In order to further investigate he electrode reaction kinetics and verify whether diffusion or adsorption is controlling factor for electron transport at the modified electrode. Figure 4 presents the effect of the scan rates on the DA and $\mathrm{AA}$ oxidation at the PANI/AgCl/GCE by CV in PBS solution at $\mathrm{pH} 4.0$ containing $1 \mathrm{mM}$ DA and 1 mM AA, respectively. A pair of redox peaks is ob- tained in each of the CV. The anodic and cathodic peak currents of DA at the modified electrode are increased linearly with the increase of the scan rates ranged from 100 to $500 \mathrm{mV} \cdot \mathrm{s}^{-1}$, as shown in Figure 4(a), indicating that direct electron transfer between DA and the modi- fied electrode. The linearity of the plots is very reason- able, with correlation coefficients of $0.9977(\mathrm{Ipc}, \mathrm{DA}(\mu \mathrm{A})=$ $-0.2636 v-13.12)$ on the cathodic current and 0.9966 $($ Ipa,DA $(\mu \mathrm{A})=0.3342 v+22.55)$ on the anodic current. 
The result implies that the electrochemical oxidation of DA at the modified electrode is dominated by a surface-adsorption controlled process [29].

The anodic peak current (Ipa,AA) of AA at PANI/ $\mathrm{AgCl} / \mathrm{GCE}$ is also proportional to the scan rates with a linear regression equation of Ipa,AA $(\mu \mathrm{A})=0.1454 v+$ $19.60(\mathrm{R}=0.9997)$, showing a typical surface-adsorption kinetics (Figure 4(b)).

The effect of $\mathrm{pH}$ on the peak potential and current were investigated by DPV in the presence of $0.1 \mathrm{mM}$ DA and $0.5 \mathrm{mM}$ AA in the $\mathrm{pH}$ range $2.0-8.0$ (the results are not shown). The results indicated that $\mathrm{AgCl}$ incorporated in PANI can catalyze the electrooxidation of DA in optimum $\mathrm{pH} 4.0$, which can obtain maximum separation of peak potential $(\triangle E \mathrm{p})$ and peak current for DA-AA, thus we choose this PBS solution as the optimum supporting electrolyte. Yan and co-workers [25] had investigated thatAgCl@ployaniline materials which showed the electrochemical selectivity to DA in $0.1 \mathrm{M}$ PBS of $\mathrm{pH} 7$ and PANI-ES to PANI-PNS occurred in $E_{2}=+0.76 \mathrm{~V}$ (vs. $\mathrm{SCE})$. However, in this study, we find that the oxidation of DA in $\mathrm{E}=0.337 \mathrm{~V}$ (vs. $\mathrm{Ag} / \mathrm{AgCl}$ ).
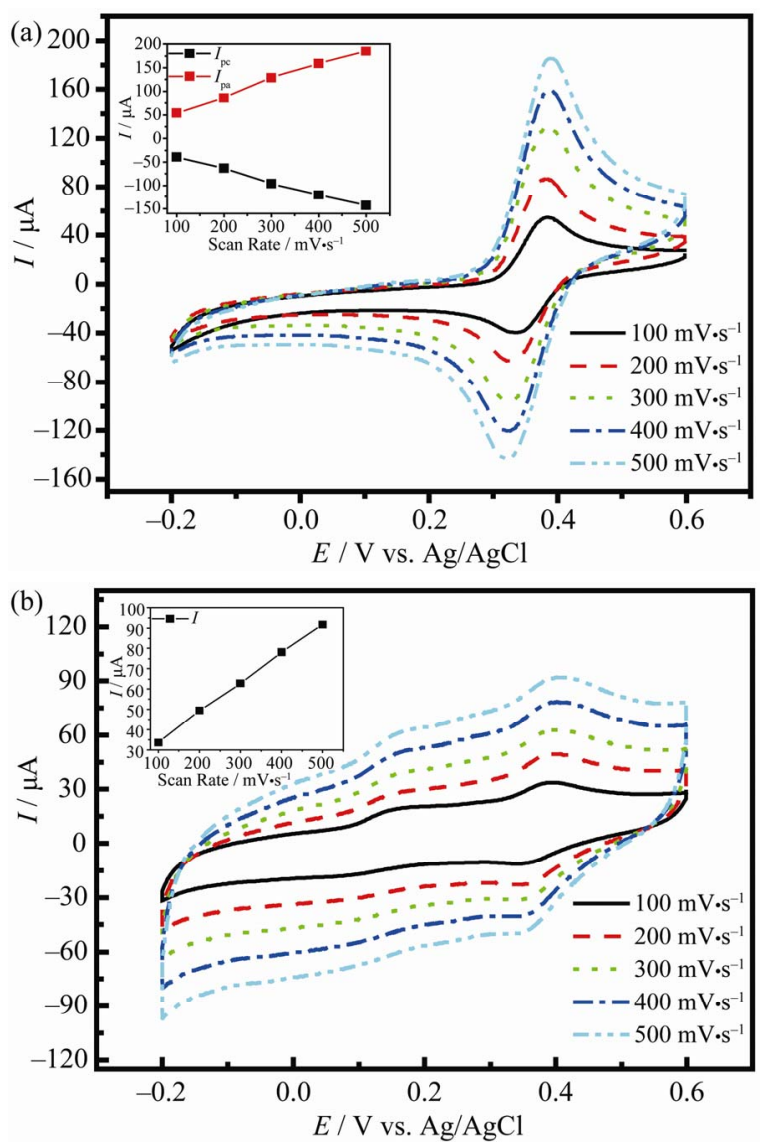

Figure 4. CVs for the oxidation of $1 \mathrm{mM} D A$ (a) and $1 \mathrm{mM}$ $\mathrm{AA}$ (b) at the PANI/AgCl/GCE in $\mathrm{pH}$ 4.0 PBS solution at different scan rates. The insert is the plots of the peak currents vs. scan rates.

\subsection{Selective Determination of $D A$ in the Presence of $\mathrm{AA}$ at the $\mathrm{PANI} / \mathrm{AgCl} / \mathrm{GCE}$}

PANI/AgCl hybrid composites were modified onto the surface of the GCE as an electrochemical sensor. The determination of DA concentration is performed with DPV technique in $0.1 \mathrm{M}$ deaerated PBS solution of $\mathrm{pH} 4.0$ in the interference from AA. It is well known that bare glassy carbon electrodes cannot resolve the oxidation peaks of DA and AA and show low sensitivity for these oxidation reactions. Some special carbon materials may be favorably altered through the modification of the electrode surface. Based on the above-mentioned demonstrations, the excellent catalytic activity of the PANI $/ \mathrm{AgCl}$ composites remarkably facilitates the electron transfer of DA at a more negative potential (see Figure 3(c)). An intrinsic property of the PANI/AgCl hybrid composites substantially differentiates the oxidation processes of DA and AA and well avoids the overlapping oxidation peaks.

Figure 5 shows the typical DPVs for the oxidation currents of DA at a fixed concentration of AA $(1 \mathrm{mM})$ at the PANI $/ \mathrm{AgCl} / \mathrm{GCE}$. The date show that two well-distinguish peaks at about 80 and $342 \mathrm{mV}$ are observed, respectively. It could be found that the DPV peak current of the DA is linearly related to its concentration within the range from $0.7 \mu \mathrm{M}$ to $6.0 \mu \mathrm{M} \mathrm{DA}$, whereas the anodic peak currents of AA is almost stable when increasing the DA concentration in the presense of AA with a concentration 1000 times higher than that of DA, revealing that the oxidation of DA and AA has no significant interference effects at the $\mathrm{PANI} / \mathrm{AgCl} / \mathrm{GCE}$. The linear regression equation is expressed as: $I \mathrm{p}, \mathrm{DA}(\mu \mathrm{A})=$ $12.7516 C_{\mathrm{DA}}(\mu \mathrm{M})+48.0274$ with a correlation coefficient of 0.9958 (the inset in Figure 5). The slope of the above equation is high to $12.7516 \mu \mathrm{A} \cdot \mu \mathrm{M}^{-1}$, which is superior

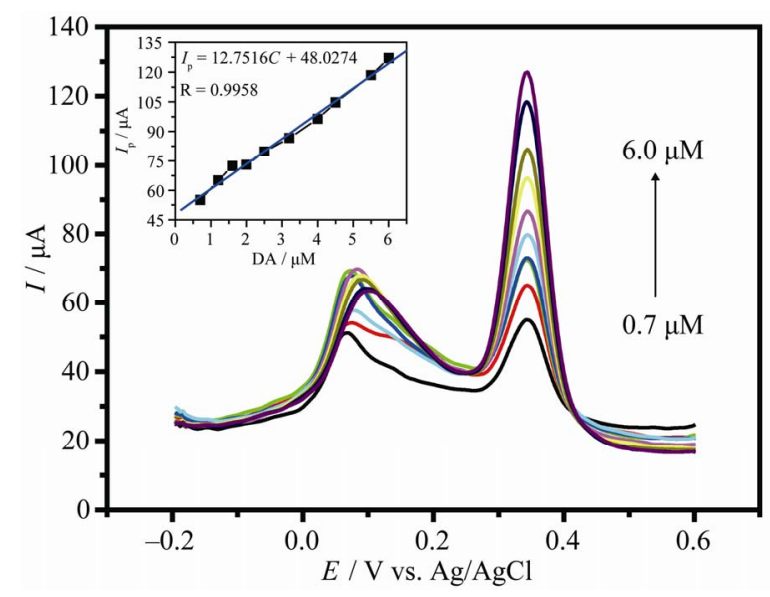

Figure 5. DPV for DA in the presence of $1 \mathrm{mM} A A$ at the PANI/AgCl/GCE in PBS at $\mathbf{p H ~ 4 . 0 . ~ D A ~ c o n c e n t r a t i o n s ~}$ (from bottom to top): $0.7,1.2,1.6,2.0,2.5,3.2,4.0,4.5,5.5$ and $6.0 \mu \mathrm{M}$. The insert is the relationship between the peak current and the concentration of DA. 
to the sensitivity response of Yan [25], and the detection limit of $5.4 \times 10^{-8} \mathrm{M}$ could be estimated $(\mathrm{S} / \mathrm{N}=3)$ [30].

These results indicate that the selective and sensitive detection of DA in the presence of high AA concentration is achieved at this modified electrode. Meanwhile, a $\triangle \mathrm{Ep}$ of $262 \mathrm{mV}$ between AA and DA allows distinguishing DA selectively by using DPV. Furthermore, the relative standard deviation ( $\mathrm{RSD} \%$ ) of the modified electrode in 10 successive scans is $1.7 \%$ for $6.0 \mu \mathrm{M}$ DA in the presence of $0.1 \mathrm{mM} \mathrm{AA}$, confirming the excellent reproducibility and stability of the $\mathrm{PANI} / \mathrm{AgCl}$ modified GCE. The results indicate that remarkable selectivity for determination of DA free from AA interference on modified electrode. A following probable reason is proposed for selectively electrochemical oxidation of DA and AA by the modified electrode. DA exists as a cation with a positive charge of amine group $(\mathrm{pKa}=8.9)$ at $\mathrm{pH} 4.0$ solution, however, AA with a $\mathrm{pKa}$ of 4.17 exists mainly as neutral form in the solution of $\mathrm{pH} 4.0$. DA has been attracted by the electrostatic force from sulfonic anion on the surface of PANI/AgCl/GCE. Therefore, DA is selectively electro-oxidized to result in the improvement of the oxidation current of DA. On the other hand, the oxidation of AA is hindered.

\section{Conclusion}

In summary, the PANI/AgCl hybrid material has been successfully used as explore the electrocatalytic propertie for the selective determination of DA in the presence of high concentrations of AA. The electrochemical tests illustrate that PANI and inorganic nanoparticle $\mathrm{AgCl}$ have a strong synergetic effect, ability to improve current, fast electron transfer kinetics and obviously decrease the overpotentials. In addition, the selective determination for DA is obtained in the range from 0.7 to $6.0 \mu \mathrm{M}$ with the detection limit of $5.4 \times 10^{-8} \mathrm{M}(\mathrm{S} / \mathrm{N}=3)$ by $\mathrm{DPV}$ under an optimum condition. The PANI/AgCl hybrid modified electrode exhibits a very low detection limit, good stability and anti-interference ability and can be utilized as a promising material for the construction of DA electrochemical sensor.

\section{Acknowledgements}

The authors wish to acknowledge the financial support from the National Natural Science Foundation of China (20876067 and 21031001) and the Fundamental Research Funds for the Central Universities (21609203).

\section{REFERENCES}

[1] C. Guzmán, G. Orozco, Y. Verde, S. Jiménez, L. A. Godínez, E. Juaristi and E. Bustos, "Hydrogen Peroxide Sensor Based on Modified Vitreous Carbon with Multiwall Carbon Nanotubes and Composites of Pt Nanoparticles-
Dopamine," Electrochimica Acta, Vol. 54, No. 6, 2009, pp. 1728-1732. doi:10.1016/j.electacta.2008.09.072

[2] Y. Zhang and J. B. Zheng, "Comparative Investigation on Electrochemical Behavior of Hydroquinone at Carbon Ionic Liquid Electrode, Ionic Liquid Modified Carbon Paste Electrode and Carbon Paste Electrode," Electrochimica Acta, Vol. 52, No. 25, 2007, pp. 7210-7216. doi:10.1016/j.electacta.2007.05.039

[3] C. S. Shan, H. E. Yang, D. X. Han, Q. X. Zhang, Ari. Ivaskac and L. Niu, "Electrochemical Determination of NADH and Ethanol Based on Ionic Liquid-Functionalized Grapheme," Biosensor Bioelectronics, Vol. 25, No. 6, 2010, pp.1504-1508. doi:10.1016/j.bios.2009.11.009

[4] J. Li and X. Q. Lin, "Simultaneous Determination of Dopamine and Serotonin on Gold Nanocluster/OveroxidizedPolypyrrole Composite Modified Glassy Carbon Electrode," Sensors and Actuators B: Chemical, Vol. 124, No. 2, 2007, pp. 486-493. doi:10.1016/j.snb.2007.01.021

[5] Z. Y. Wang, S. N. Liu, P. Wu and C. X. Cai, "Detection of Glucose Based on Direct Electron Transfer Reaction of Glucose Oxidase Immobilized on Highly Ordered Polyaniline Nanotubes," Analytical Chemical, Vol. 81, No. 4, 2009, pp. 1638-1645. doi:10.1021/ac802421h

[6] M. A. Dennis, K. Michael, M. M. Khodabakhsh, N. Stuart and W. Michael, "Electrochemical Activation of Carbon Electrodes in Base: Minimization of Dopamine Adsorption and Electrode Capacitance," Analytical Chemical, Vol. 61, No. 23, 1989, pp. 2603-2608. doi:10.1021/ac00198a004

[7] B. J. Christopher, P. M. Jennifer and V. B. Jill, "Review: Carbon Nanotube Based Electrochemical Sensors for Biomolecules," Analytica Chimica Acta, Vol. 662, No. 2, 2010, pp. 105-127. doi:10.1016/j.aca.2010.01.009

[8] C. Martin, “The Parkinson's Puzzle: New Developments in Our Understanding of Parkinson's Disease Have Generated a Number of Promising New Treatments for This Disabling Condition," Chemistry in Britian, Vol. 34, No. 9, 1998, pp. 40-42.

[9] A. Heinz, H. Przuntek, G. Winterer and A. Pietzcker, "Clinical Aspects and Follow-Up of Dopamine-Induced Psychoses in Continuous Dopaminergic Therapy and Their Implications for the Dopamine Hypothesis of Schizophrenic Symptoms," Der Nervenarzt, Vol. 66, No. 9, 1995, pp. 662-669.

[10] R. M. Wightman, L. J. May and A. C. Michael, "Detection of Dopamine Dynamics in the Brain," Analytical Chemical, Vol. 60, No. 13, 1988, pp. 769-779. doi:10.1021/ac00164a001

[11] G. Y. Jin, Y. Z. Zhang and W. X. Cheng, "Poly(p-Aminobenzene Sulfonic Acid)-Modified Glassy Carbon Electrode for Simultaneous Detection of Dopamine and Ascorbic Acid," Sensors and Actuators B: Chemical, Vol. 107, No. 2, 2005, pp. 528-534. doi:10.1016/i.snb.2004.11.018

[12] H. S. Wang, T. H. Li, W. L. Jia and H. Y. Xu, "Highly Selective and sensitive Determination of Dopamine Using a Nafion/Carbon Nanotubes coated Poly(3-Methylthiophene) Modified Electrode," Biosensors and Bioelectronics, Vol. 22, No. 5, 2006, pp. 664-669.

doi:10.1016/i.bios.2006.02.007 
[13] T. Selvaraju and R. Ramaraj, "Simultaneous Determination of Ascorbic Acid, Dopamine and Serotonin at Poly (Phenosafranine) Modified Electrode," Electrochemistry Communications, Vol. 5, No. 8, 2003, pp. 667-672. doi:10.1016/S1388-2481(03)00151-6

[14] Y. X. Li and X. Q. Lin, "Simultaneous Electroanalysis of Dopamine, Ascorbic Acid and Uric Acid by Poly (Vinyl Alcohol) Covalently Modified Glassy Carbon Electrode," Sensors and Actuators B: Chemical, Vol. 115, No. 1, 2006, pp. 134-139. doi:10.1016/j.snb.2005.08.022

[15] H. R. Zare, N. Nasirizadeh and M. M. Ardakani, "Electrochemical Properties of a Tetrabromo-Pbenzoquinone Modified Carbon Paste Electrode. Application to the Simultaneous Determination of Ascorbic Acid, Dopamine and Uric Acid," Journal of Electroanalytical Chemistry, Vol. 577, No. 1, 2005, pp. 25-33. doi:10.1016/i.jelechem.2004.11.010

[16] S. Yunus, A. Attout and P. Bertrand, "Controlled Aniline Polymerization Strategies for Polyaniline Micro- and Nano Self-Assembling into Practical Electronic Devices" Langmuir, Vol. 25, No. 3, 2009, pp. 1851-1854. doi:10.1021/la803034q

[17] J. Jang, J. Ha and J. Cho, "Fabrication of Water-Dispersible Polyaniline-Poly(4-Styrenesulfonate) Nanoparticles for Inkjet-Printed Chemical-Sensor Applications," Advanced Materidals, Vol. 19, No. 13, 2007, pp. 17721775. doi:10.1002/adma.200602127

[18] Y. Qiao, S. J. Bao, C. M. Li, X. Q. Cui, Z. S. Lu and J. Guo, "Nanostructured Polyanifine/Titanium Dioxide Composite Anode for Microbial Fuel Cells.," Acs Nano, Vol. 2, No. 1, 2008, pp.113-119. doi:10.1021/nn700102s

[19] Z. M. Zhang, J. Sui, L. J. Zhang, M.X. Wan, Y. Wei and L. M. Yu, "Synthesis of Polyaniline with a Hollow, Octahedral Morphology by Using a Cuprous Oxide Template," Advanced Materidals, Vol. 17, No. 23, 2005, pp. 2854-2857. doi:10.1002/adma.200501114

[20] J. Han, G. Song and R. Guo, "Nanostructure-Based LeafLike Polyaniline in the Presence of an Amphiphilic Triblock Copolymer," Advanced Materidals, Vol. 19, No. 19, 2007, pp. 2993-2999. doi:10.1002/adma.200602635

[21] T. Yin, W. Wei and J. Zeng, "Selective Detection of Dopamine in the Presence of Ascorbic Acid by Use of Glassy-Carbon Electrodes Modified with Both Polyaniline Film and Multi-Walled Carbon Nanotubes with Incorporated Beta-Cyclodextrin," Analytical and Bioanalytical Chemistry, Vol. 386, No. 7-8, 2006, pp. 2087-2094. doi:10.1007/s00216-006-0845-Z

[22] S. R. Ali, Y. F. Ma, R. R. Parajuli, Y. Balogun, W. Y. C.
Lai and H. X. He, "A Nonoxidative Sensor Based on a Self-Doped Polyaniline/Carbon Nanotube Composite for Sensitive and Selective Detection of the Neurotransmitter Dopamine," Analytical Chemical, Vol. 79, No. 6, 2007, pp. 2583-2587. doi:10.1021/ac0620680

[23] V. Zucolotto, M. Ferreira, M. R. Cordeiro, C. J. L. Constantino, W. C. Moreira and O. N. Oliveira, "Nanoscale Processing of Polyaniline and Phthalocyanines for Sensing Applications," Sensors and Actuators B: Chemical, Vol. 113, No. 2, 2006, pp. 809-815. doi:10.1016/j.snb.2005.03.114

[24] K. Pihel, Q. D. Walker and R. M. Wightman, "Overoxidized Polypyrrole-Coated Carbon Fiber Microelectrodes for Dopamine Measurements with Fast-Scan Cyclic Voltammetry," Analytical Chemical, Vol. 68, No. 13, 1996, pp. 2084-2089. doi:10.1021/ac960153y

[25] W. Yan, X. M. Feng, X. J. Chen, X. H. Li and J. J. Zhu, "A Selective Dopamine Biosensor Based on

AgCl@polyaniline Core-Shell Nanocomposites," Bioelectrochemistry, Vol. 72, No. 1, 2008, pp. 21-27. doi:10.1016/j.bioelechem.2007.07.003

[26] X. M. Feng, Y. Liu, C. L. Lu, W. H. Hou and J. J. Zhu, "One-Step Synthesis of Agcl/Polyaniline Core-Shell Composites with Enhanced Electroactivity," Nanotechnology, Vol. 17, No. 14, 2006, pp. 3578-3583. doi:10.1088/0957-4484/17/14/037

[27] A. Rahy and D. J. Yang, "Synthesis of Highly Conductive Polyaniline Nanofibers," Materials Letters, Vol. 62, No. 28, 2008, pp. 4311-4314 doi:10.1016/j.matlet.2008.06.057

[28] S. L. Zhou, S. S. Mo, W. J. Zou, F. P. Jiang, T. X. Zhou and D. S.Yuan, "Preparation of Polyaniline/2-Dimensional Hexagonal Mesoporous Carbon Composite for Supercapacitor," Synthetic Metals, Vol. 161, No. 15-16, 2011, pp. 1623-1628 doi:10.1016/j.synthmet.2011.05.028

[29] D. Zheng, J. S. Ye, L. Zhou, Y. Zhang and C. Z. Yu, "Simultaneous Determination of Dopamine, Ascorbic Acid, and Uric Acid on Ordered Mesoporous Carbon/Nafion Composite Film," Journal of Electroanalytical Chemistry, Vol. 625, No. 1, 2009, pp. 82-87. doi:10.1016/j.jelechem.2008.10.012

[30] J. W. Mo and B. Ogorevc, "Simultaneous Measurement of Dopamine and Ascorbate at Their Physiological Levels Using Voltammetric Microprobe Based on Overoxidized Poly(1,2-Phenylenediamine)-Coated Carbon Fiber," Analytical Chemical, Vol. 73, No. 6, 2001, pp. 1196-1202. doi:10.1021/ac0010882 